\title{
Theoretical study of the elasticity, mechanical behavior, electronic structure, interatomic bonding, and dielectric function of an intergranular glassy film model in prismatic $\beta-\mathrm{Si}_{3} \mathrm{~N}_{4}$
}

\author{
W. Y. Ching, ${ }^{1}$ Paul Rulis, ${ }^{1}$ Lizhi Ouyang, ${ }^{2}$ Sitaram Aryal, ${ }^{1}$ and Anil. Misra ${ }^{3}$ \\ ${ }^{1}$ Department of Physics, University of Missouri-Kansas City, Kansas City, Missouri 64110, USA \\ ${ }^{2}$ Department of Physics and Mathematics, Tennessee State University, Nashville, Tennessee 37221, USA \\ ${ }^{3}$ Department of Civil, Environmental, and Architectural Engineering, University of Kansas, Lawrence, Kansas 66045, USA
}

(Received 31 March 2010; revised manuscript received 29 May 2010; published 24 June 2010)

\begin{abstract}
Microstructures such as intergranular glassy films (IGFs) are ubiquitous in many structural ceramics. They control many of the important physical properties of polycrystalline ceramics and can be influenced during processing to modify the performance of devices that contain them. In recent years, there has been intense research, both experimentally and computationally, on the structure and properties of IGFs. Unlike grain boundaries or dislocations with well-defined crystalline planes, the atomic scale structure of IGFs, their fundamental electronic interactions, and their bonding characteristics are far more complicated and not well known. In this paper, we present the results of theoretical simulations using $a b$ initio methods on an IGF model in $\beta$ - $\mathrm{Si}_{3} \mathrm{~N}_{4}$ with prismatic crystalline planes. The 907-atom model has a dimension of $14.533 \AA \times 15.225 \AA$ $\times 47.420 \AA$. The IGF layer is perpendicular to the $z$ axis, $16.4 \AA$ wide, and contains $72 \mathrm{Si}, 32 \mathrm{~N}$, and $124 \mathrm{O}$ atoms. Based on this model, the mechanical and elastic properties, the electronic structure, the interatomic bonding, the localization of defective states, the distribution of electrostatic potential, and the optical dielectric function are evaluated and compared with crystalline $\beta-\mathrm{Si}_{3} \mathrm{~N}_{4}$. We have also performed a theoretical tensile experiment on this model by incrementally extending the structure in the direction perpendicular to the IGF plane until the model fully separated. It is shown that fracture occurs at a strain of $9.42 \%$ with a maximum stress of $13.9 \mathrm{GPa}$. The fractured segments show plastic behavior and the formation of surfacial films on the $\beta-\mathrm{Si}_{3} \mathrm{~N}_{4}$. These results are very different from those of a previously studied basal plane model [J. Chen et al., Phys. Rev. Lett. 95, 256103 (2005)] and add insights to the structure and behavior of IGFs in polycrystalline ceramics. The implications of these results and the need for further investigations are discussed.
\end{abstract}

DOI: 10.1103/PhysRevB.81.214120

PACS number(s): 68.35.-p, 81.05.Je, 71.23.An, 78.66.Sq

\section{INTRODUCTION}

Microstructures such as intergranular glassy films (IGFs) are ubiquitous in many structural ceramics such as silicon nitrides, silicon carbides, and alumina. Within the last decade or so there has been intense research, both experimentally and computationally, on the structure and properties of IGFs since they control many of the most important physical properties in polycrystalline ceramics, especially the mechanical properties. ${ }^{1-5}$ Unlike grain boundaries or dislocations with well-defined crystalline phases, the atomic scale structure of IGFs is far more complicated. Indeed, some consider IGFs to be the most complex member of a family of grain boundaries in polycrystalline ceramics ${ }^{6,7}$ and further its origin has been suggested to be similar to the surfacial films in some crystals. ${ }^{8,9}$

There have been several theories related to the formation and thermodynamic stability of IGFs in ceramics. ${ }^{10-14}$ However, both the thermodynamic model based on Cahn-Hillard theory ${ }^{15}$ or its extension and the interface force balance model of Clarke ${ }^{10}$ lacks the atomistic description of the IGF. Direct experimental evidence that supports or refutes these theories is hard to obtain because of the complexity of the IGF structure and the many unknown variables involved in the formation of IGFs. For example, a small change in the IGF composition and structure could drastically affect the interface strength and mechanical properties. ${ }^{16}$ Experimental investigations based on high-resolution transmission electron microscopy (HRTEM), electron energy-loss spectroscopy, and energy-dispersive $\mathrm{x}$-ray spectroscopy have significantly contributed to the early understanding of the structures of IGFs in polycrystalline ceramics. ${ }^{17-22}$ However, limitations in instrumental resolution and other abilities have hindered the attainment of a deeper understanding of the mechanisms related to the formation of IGFs and how they interact with bulk materials. In particular, HRTEM faces some difficulty with the noncolumnar structure of the glassy region. Furthermore, phonon scattering or thermodiffuse scattering is always present and the ability of experimental measurements to isolate such scattering is a daunting task. Yet, with the rapid advancement of instrumentation, such as aberration corrected scanning transmission electron microscopy, many exciting new results on the structure of IGFs have been reported in recent years. ${ }^{23-27}$

In this regard, computational simulations on well constructed IGF models will be extremely valuable. They can provide much needed insight into the atomic scale interactions which are particularly difficult to obtain from laboratory experiments. Understanding of the fundamental physical properties of IGFs (e.g., elastic properties, mechanical strength, structure and composition, chemical bonding, electronic and dielectric properties, and space-charge distribution) is important if we are going to comprehend the mechanism by which we can make polycrystalline ceramics tough and creep resistant, and to extend their range of application with improved properties. Theoretically, the ability to reveal the complicated structure-property relationships and provide quantitative, not qualitative, results is severely inhibited by 
the absence of well characterized IGF models and by the limitations inherent in performing accurate calculations. Yoshiya et al. predicted that the IGF thickness is about $7 \AA$ using classical molecular-dynamics (MD) simulations on large models of several thousand atoms. ${ }^{28,29} \mathrm{MD}$ simulations using more accurate multiatom potentials have also been used to create IGF models. ${ }^{30,31}$ Simulations based on the reaction force-field model with many additional calibrated parameters derived from chemical bonding considerations in small molecules have also been used to model IGF structures. ${ }^{32}$ Such MD simulations have provided us with good insights into the structure, composition, and stability of IGFs. However, to understand the details of the interplay between the electronic structure and measurable physical properties, a full quantum-mechanical description of the interaction is necessary.

Considerable advancements in ab initio methods based on density-functional theory (DFT) (Refs. 33 and 34) have emerged in recent years fueled by both the availability of supercomputing resources and the development of efficient computational techniques. We have previously demonstrated that $a b$ initio simulations can be successfully applied to IGF models in $\beta-\mathrm{Si}_{3} \mathrm{~N}_{4}$ to yield a wealth of previously unknown information. ${ }^{35-38}$ The model we previously constructed has 798 atoms sandwiched between the basal planes of $\beta-\mathrm{Si}_{3} \mathrm{~N}_{4}$ (referred to as the basal IGF model). The basal IGF model, with its defective structure at the glass/crystal interface, can be considered as a kind of diffuse interface model where the boundary between the crystalline part and the glass part is not perfectly sharp. Results from theoretical tensile experiments on both the pure [no rare-earth (RE) additives] and Y-doped model indicate that the behavior of the mechanical deformation is highly nonlinear in clear violation of the Cauchy Born theory routinely accepted by the engineering community. ${ }^{36}$ It was shown that the addition of RE elements, such as $\mathrm{Y}$, enhances the mechanical properties in agreement with experimental observations. ${ }^{16,39-41}$ The detailed source of this effect was identified by a quantum-mechanical analysis of the bonding of characteristics of $Y$ within the IGF. This is one of the main reasons for using RE oxides as sintering aids in the processing of high-density polycrystalline silicon nitrides. Other researchers have used smaller clusters to model the interface between the crystalline plane and glassy structure to ascertain the stability of the interface and the local geometry of the RE element. ${ }^{42,43}$

In this paper, we report theoretical results on the physical properties of an IGF model in $\beta-\mathrm{Si}_{3} \mathrm{~N}_{4}$ with prismatic crystalline $[10 \overline{1} 0]$ planes. The present results on the prismaticIGF model are very different from those of the basal model studied previously. ${ }^{36}$ This difference is rooted in the differences in atomic scale structure between the crystalline and glassy phases, underscoring the importance of characterizing and studying individual IGF models instead of attempting sweeping generalization. The prismatic model is a much more realistic model than the basal model since many HRTEM pictures of silicon nitride show that the needlelike polycrystalline grains grow more easily in the [001] direction indicating that it is easier to form an IGF with prismatic planes. We start with the description of a carefully con- structed and fully relaxed model that is then used to obtain the mechanical and elastic properties, the electronic structure, the interatomic bonding, the localization of defective states, the distribution of electrostatic potential, and the optical dielectric function. These results are then compared with crystalline $\beta$-Si ${ }_{3} \mathrm{~N}_{4}$. We have also performed theoretical tensile experiments on this prismatic model by incrementally extending the structure in the direction perpendicular to the IGF plane until the model fractures. Some preliminary results on the tensile experiment have been reported previously. ${ }^{44}$ The present paper contains a much more detailed analysis with improved accuracy of the numerical data and also a critical analysis of the postfracture structure which mimics that of surfacial films. The presence of residual stress after fracture can strongly affect the mechanical properties and the lifetime performance of devices containing polycrystalline ceramic components.

In the next section, Sec. II, we describe the methods used in the simulation studies and in the analysis of physical properties. In Sec. III, the procedures involved in the construction of the prismatic-IGF model and its structure and composition are fully described. The main results for various physical properties of the prismatic-IGF model are presented in Sec. IV. We then present the results of the theoretical uniaxial tensile experiment and discuss its postfailure behavior. The implications of these results are further discussed in Sec. VI. The paper ends with brief conclusions together with a logical projection of the next step in the fundamental investigations of microstructures in ceramics.

\section{METHOD OF SIMULATION AND ANALYSIS}

We used two well established $a b$ initio methods for the simulation and analysis of the IGF model. The Vienna $a b$ initio simulation package (VASP) (Refs. 45 and 46) is used for structural relaxation and tensile experiment as well as for mechanical and elastic properties calculations. The orthogonalized linear combination of atomic orbitals method (OLCAO) (Ref. 47) using the local-density approximation (LDA) is used for electronic structure, bonding, and optical analysis based on the VASP-relaxed structures. The combination of these two $a b$ initio methods has proven extremely effective especially for large complex systems as demonstrated in several recent publications including the studies of the basal IGF model. Other groups have also used a combination of two or several methods with different strengths to investigate complex structures. ${ }^{42,48}$

For VASP calculations including the theoretical tensile experiment (see Sec. V), we used Vanderbilt ultrasoft pseudopotentials $^{49}$ with the LDA for evaluating the exchange-correlation potential and a relatively high-energy cutoff of $500 \mathrm{eV}$. A criterion of $10^{-5} \mathrm{eV}$ for electronic convergence and $10^{-2} \mathrm{eV} / \mathrm{A}$ for force convergence was adopted. The stress level for the equilibrium structure is below $0.1 \mathrm{GPa}$. The IGF model has a cell volume sufficiently large so that only one $k$ point at $(0,0,0)$ was used for Brillouin-zone (BZ) integration.

In the OLCAO calculation we used a full basis (FB) set of atomic orbitals of $\mathrm{Si}(1 s, 2 s, 3 s, 4 s, 2 p, 3 p, 4 p, 3 d)$, 
$\mathrm{N}(1 s, 2 s, 3 s, 2 p, 3 p)$, and $\mathrm{O}(1 s, 2 s, 2 p, 3 s, 3 p)$ where italicized orbitals are orthogonalized out of the secular equation. For obtaining the self-consistent field potential, a FB is more than sufficient. The secular equation of dimension 9111 $\times 9111$ is diagonalized to obtain all energy eigenvalues and wave functions for properties analysis. In the case of the optical dielectric function calculation, it is desirable to have an even larger basis set so an additional shell of atomic orbitals was added to the FB set. This extended basis makes the dimension of the final secular equation become 15090 $\times 15090$.

Since a basis of atomic orbitals is used in the OLCAO method, it is very convenient to obtain the effective charge $Q_{\alpha}^{*}$ on each atom and the bond order (BO) $\rho_{\alpha \beta}$ between each pair of atoms using the Mulliken scheme, ${ }^{50}$

$$
\begin{gathered}
Q_{\alpha}^{*}=\sum_{i} \sum_{n, o c c} \sum_{j, \beta} c_{i \alpha}^{* n} c_{j \beta}^{n} s_{i \alpha, j \beta}, \\
\rho_{\alpha \beta}=\sum_{n, o c c} \sum_{i, j} C_{i \alpha}^{* n} C_{j \beta}^{n} S_{i \alpha, j \beta} .
\end{gathered}
$$

In Eqs. (1) and (2), $i$ and $j$ label the orbital quantum numbers of atoms $\alpha$ and $\beta$ while $n$ is the band index. $S_{i \alpha, j \beta}$ is the overlap matrix between OLCAO Bloch sums and $C_{j \beta}^{n}$ is an eigenvector coefficient of the wave function. Because the Mulliken scheme works best when the basis functions are localized, a minimal basis (MB) set which includes only occupied atomic orbitals is used for effective charge and bond order calculations. The MB typically has one shell less than the FB.

\section{PRISMATIC-IGF MODEL}

The construction of the prismatic-IGF model in this paper is entirely different from the construction of the basal IGF model. ${ }^{35}$ In the basal model we used classical MD with multiatom potentials to obtain the initial structure and then later relaxed it using VASP. The basal model contains defective structures at the IGF/crystalline interface as explained in Ref. 35. Also, the convergence criteria used in the VASP relaxation for the basal model were less stringent. We used a protocol to construct the prismatic model, with an IGF of chosen chemical composition, based on the idea of simulated annealing. The prismatic-IGF model was generated using a controlled combination of three computational packages in order to simultaneously attain the necessary accuracy while maintaining sufficient computational efficiency at every stage in the process. An initial fluidlike phase consisting of a mixture of $\mathrm{Si}, \mathrm{N}$, and $\mathrm{O}$ atoms of appropriate composition ratios and density was generated using the MOLDY package. ${ }^{51}$ The simulated annealing was carried out in two phases using the SIESTA $\operatorname{code}^{52}$ based on pseudopotential method with LDA for exchange correction. All simulations using SIESTA are performed under constant volume conditions. For hightemperature simulations, single zeta basis sets were employed. For lower temperature simulations, double zeta polarized basis sets were used. In the first phase of simulation, the IGF layer was sandwiched between two crystalline layers of $\beta-\mathrm{Si}_{3} \mathrm{~N}_{4}$ with a prismatic orientation. The atoms in the crystalline layers were fixed while the gas phase mixture was quickly quenched from very high temperature of $8000 \mathrm{~K}$ to high temperature of $3000 \mathrm{~K}$. The model was then annealed at $3000 \mathrm{~K}$ for 1 ps. Such simulated annealing may be repeated several times until a reasonable chemical bonding network is formed below the melting temperature of $1000 \mathrm{~K}$. The radial pair distribution functional (RPDF) was calculated and monitored to trace the local bonding changes. In phase II, more crystalline layers were added to the original two crystalline layers to form the bulk region of the IGF model with periodic boundary conditions. The goal of phase II was to equalize the IGF/crystalline interface. In step one of phase II, atoms of the crystalline layers near the glassy region were also allowed to move. Multiple rounds of simulated annealing were carried out at gradually decreasing temperatures of modest range to prevent the atoms from diffusing into the crystalline bulk. Finally, all atoms including those of the bulk crystalline part were allowed to move. Several rounds of simulated annealing were carried out in a narrow range around the IGF melting temperature. The stress of the model was carefully monitored and the thickness of the IGF layer was adjusted to ensure that the stress level of the sandwiched IGF layer matches that of the bulk crystal part and that the interface structure is similar to what is experimentally observed. ${ }^{23,24}$ At last, the whole model was cooled to room temperature and equalized at $300 \mathrm{~K}$ for $1 \mathrm{ps}$. A snapshot of the model at the end of equalization was then taken to be the initial configuration for the prismatic-IGF model which maintains periodic boundary conditions in three dimensions.

This initial prismatic-IGF model was then further relaxed using VASP with high accuracy by slightly varying the thickness of the glassy region. The one with the lowest total energy was then selected as the final model. This final model contains 907 atoms of which 228 atoms are in the IGF region (72 Si, $32 \mathrm{~N}$, and $124 \mathrm{O}$ ) and the other 679 atoms are in the crystalline bulk region ( $299 \mathrm{Si}$ and $380 \mathrm{~N}$ ). The model has a dimension of $14.533 \AA \times 15.225 \AA \times 47.420 \AA$ and an IGF width of about $1.64 \mathrm{~nm}$ as shown in Figs. 1(a) and 1(b) in two different orientations. The exact boundary between the crystal part and the glassy part in the model involves some uncertainty. We defined the boundary as the line between the Si layer and the $\mathrm{N}$ layer closest to the IGF in the original crystal before joining it with the glassy portion. The $\mathrm{N}$ ions in that layer are counted as IGF ions together with the $\mathrm{Si}, \mathrm{O}$, and $\mathrm{N}$ ions from the glass phase. Figure 1(c) shows the RPDF of the glassy region in the prismatic model. This RPDF is in close agreement with experimental data ${ }^{53}$ including the very broad peaks at 4.3 and $5.1 \AA$ in Fig. 1(c), even though the samples used in the experiment contain RE elements as part of the sintering aid.

When experimentally characterizing an IGF structure it is customary to specify the ratio of the ions to the total number of anions in the glassy region. In the present model, these ratios are $\mathrm{Si} /(\mathrm{N}+\mathrm{O})=0.46, \mathrm{~N} /(\mathrm{N}+\mathrm{O})=0.21$, and $\mathrm{O} /(\mathrm{N}$ $+\mathrm{O})=0.79$; which are typical values, consistent with those observed in real samples. ${ }^{21}$ There are indications that the mechanical strength and perhaps other physical properties of polycrystals with IGFs could depend on the $\mathrm{N}$ content within the IGF. This issue will be further discussed later.

In crystalline $\beta-\mathrm{Si}_{3} \mathrm{~N}_{4}$, all $\mathrm{Si}$ atoms are tetrahedrally bonded to four $\mathrm{N}$ and each $\mathrm{N}$ atom is bonded to three $\mathrm{Si}$ in an 

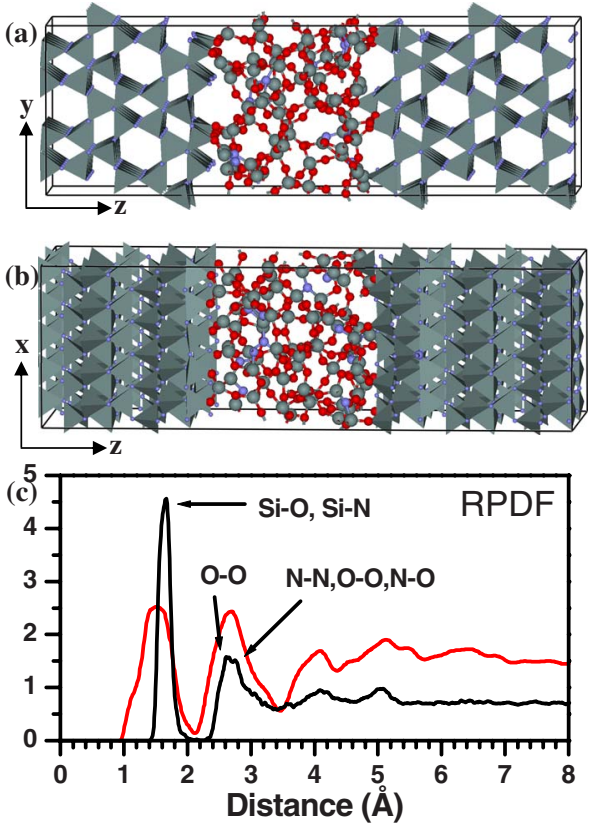

FIG. 1. (Color online) Ball and stick models of the 907-atom prismatic model. The crystalline region is shown as $\mathrm{SiN}_{4}$ tetrahedrons. Si (gray), N (blue), and O (red). (a) Viewed in the $y$ direction and (b) viewed in the $x$ direction. (c) The radial pair distribution function of the IGF portion of the model. The experimental curve (red line) is from Ref. 53.

approximate planar fashion. In the IGF region, the bonding between $\mathrm{Si}$ and the anions ( $\mathrm{O}$ and $\mathrm{N}$ ) can be quite different. We have identified the local bonding configurations for all the atoms in the IGF region and at the boundaries with the crystal part using bond lengths in the crystalline phase as the criterion. We found that the majority of the $\mathrm{Si}$ atoms are fourfold bonded to either $4 \mathrm{O}\left(\mathrm{Si}-\mathrm{O}_{4}\right)$ or $3 \mathrm{O}$ and $1 \mathrm{~N}$ $\left(\mathrm{Si}-\mathrm{NO}_{3}\right)$. There are also a few other fourfold bonded $\mathrm{Si}$ atoms in the form of Si- $\mathrm{N}_{4}, \mathrm{Si}-\mathrm{N}_{2} \mathrm{O}_{2}$, and $\mathrm{Si}-\mathrm{N}_{3} \mathrm{O}$. There are eight threefold bonded $\mathrm{Si}\left(\mathrm{Si}-\mathrm{NO}_{2}\right.$ and $\left.\mathrm{Si}-\mathrm{N}_{2} \mathrm{O}\right)$ and one fivefold bonded $\mathrm{Si}\left(\mathrm{Si}-\mathrm{O}_{5}\right)$ which can be considered as a defec- tive $\mathrm{Si}$. For the anions, there are seven $\mathrm{N}$ atoms that are twofold bonded to $\mathrm{Si}$ and two $\mathrm{O}$ atoms that are threefold bonded to $\mathrm{Si}$ in addition to $1 \mathrm{O}$ having a dangling bond (O-Si). All Si-O and $\mathrm{Si}-\mathrm{N}$ bond distances are very reasonable with only a few bonds as large as $1.87 \AA$. A remarkable point of the model is that unlike the models derived from reaction force-field $\mathrm{MD},{ }^{32}$ there are no bonds between anions $(\mathrm{O}-\mathrm{N}, \mathrm{N}-\mathrm{N}$, and $\mathrm{O}-\mathrm{O})$. Thus, the structure of the IGF region is close to that of an ideal random network. The different bonding configurations are part of the glassy structures and they introduce significant variations in the fundamental electronic structure especially for states near the band gap. They are also intimately related to the mechanical properties of the IGF model that will be elaborated on later. The structural characteristics of the prismatic model are listed in Table I.

There has been considerable debate about the interface structure of the IGF and bulk crystals. In the present model, this interface boundary is quite sharp. This is in agreement with HRTEM images that show such sharp boundaries. ${ }^{23-26}$ However, there exist a few underbonded $\mathrm{Si}$, and the $\mathrm{N}$ atoms show a tendency to be underbonded, although it is known that threefold bonded $\mathrm{Si}$ atoms do occur in some crystalline phases. ${ }^{54}$ In contrast, the basal model in the earlier studies had a rather blurred interface due to the existence of defective structures within the first three crystalline layers next to the IGF. ${ }^{35}$ On the other hand, the so-called diffuse interface model argues that the crystal/IGF boundary should not be sharp. ${ }^{55}$ Obviously, a clear distinction between a diffuse interface model and that with a more abrupt sharp interface cannot be made based on the study of one or two such models.

\section{RESULTS OF THE EQUILIBRIUM MODEL}

\section{A. Mechanical and elastic properties}

While the structures and composition of different IGF models have been theoretically studied by several groups in recent years, $a b$ initio calculation of the mechanical properties of a realistic model has, to our knowledge, not been

TABLE I. Structure and composition of the prismatic-IGF model. The $z$ direction of the model is perpendicular to the IGF layer.

\begin{tabular}{ll}
\hline \hline Cell dimension & $a=14.533 \AA, b=15.225 \AA$, and $c=47.420 \AA$ \\
Width of IGF region & $16.4 \AA$ \\
Total number of atoms & 907 \\
Bulk crystal & $679(299 \mathrm{Si}, 380 \mathrm{~N})$ \\
IGF & $228(72 \mathrm{Si}, 32 \mathrm{~N}, 124 \mathrm{O})$ \\
Composition ratio & \\
of atoms in the IGF & $\mathrm{Si} /(\mathrm{N}+\mathrm{O})=0.46, \mathrm{~N} /(\mathrm{N}+\mathrm{O})=0.21$, and $\mathrm{O} /(\mathrm{N}+\mathrm{O})$ \\
relative to total anions & $=0.79$ \\
Bonding configurations in IGF & Bulk crystal: $\mathrm{Si}-\mathrm{N}_{4}$ and $\mathrm{N}-\mathrm{Si}_{3}$ \\
IGF & Major configurations: $\mathrm{Si}-\mathrm{O}_{4}$ and $\mathrm{Si}_{-} \mathrm{NO}_{3} ;$ \\
& Minor configurations: $\mathrm{Si}-\mathrm{N}_{4}, \mathrm{Si}_{-} \mathrm{N}_{2} \mathrm{O}_{2}$, and \\
& $\mathrm{Si}-\mathrm{N}_{3} \mathrm{O}$. \\
& Defective configurations: $\mathrm{Si}-\mathrm{NO}_{2}, \mathrm{Si}_{-} \mathrm{N}_{2} \mathrm{O}$, and \\
& Si-O $\mathrm{O}_{5}$ \\
\hline \hline
\end{tabular}


TABLE II. Elastic and mechanical properties of the prismatic-IGF model compared with the values for the crystalline $\beta$-Si $\mathrm{N}_{4}$ (in parenthesis) in unit of GPa. Please note the difference in the Cartesian directions $\mathrm{x}, \mathrm{y}, \mathrm{z}$ for the IGF model (z axis perpendicular to IGF layer) and the $\beta-\mathrm{Si}_{3} \mathrm{~N}_{4}$ crystal ( $z=c$-axis of the hexagonal cell).

\begin{tabular}{|c|c|c|c|c|c|c|c|c|c|}
\hline \multirow[t]{2}{*}{ IGF model } & $\mathrm{C}_{11}$ & $\mathrm{C}_{22}$ & $\mathrm{C}_{33}$ & $\mathrm{C}_{12}$ & $\mathrm{C}_{13}$ & $\mathrm{C}_{23}$ & $\mathrm{C}_{44}$ & $\mathrm{C}_{55}$ & $\mathrm{C}_{66}$ \\
\hline & 411.8 & 322.3 & 305.7 & 76.7 & 75.0 & 119.8 & 96.3 & 86.7 & 88.9 \\
\hline$\beta-\mathrm{Si}_{3} \mathrm{~N}_{4}$ & $\mathrm{C}_{11}$ & $\mathrm{C}_{22}$ & $\mathrm{C}_{33}$ & $\mathrm{C}_{12}$ & $\mathrm{C}_{13}$ & $\mathrm{C}_{23}$ & $\mathrm{C}_{44}$ & $\mathrm{C}_{55}$ & $\mathrm{C}_{66}$ \\
\hline Calc. & 431.3 & 431.3 & 551.7 & 178.1 & 108.0 & & 104.5 & & 126.6 \\
\hline Expt. $^{\mathrm{a}}$ & $433 \pm 3$ & $433 \pm 3$ & $574 \pm 3$ & $195 \pm 8$ & $127 \pm 5$ & & $108 \pm 2$ & & $119 \pm 4$ \\
\hline \multirow[t]{3}{*}{ IGF portion } & $\mathrm{C}_{11}$ & $\mathrm{C}_{22}$ & $\mathrm{C}_{33}$ & $\mathrm{C}_{12}$ & $\mathrm{C}_{13}$ & $\mathrm{C}_{23}$ & $\mathrm{C}_{44}$ & $\mathrm{C}_{55}$ & $\mathrm{C}_{66}$ \\
\hline & 161.5 & 127.3 & 81.0 & 20.7 & 18.2 & 15.5 & 54.9 & 61.0 & 42.1 \\
\hline & & & \multicolumn{2}{|c|}{ IGF model } & \multicolumn{2}{|c|}{$\beta-\mathrm{Si}_{3} \mathrm{~N}_{4}$} & \multicolumn{2}{|c|}{ IGF portion } & \\
\hline \multicolumn{2}{|c|}{ Bulk modulus $K$} & & \multicolumn{2}{|c|}{175.4} & \multicolumn{2}{|c|}{244.2} & \multicolumn{2}{|c|}{51.0} & \\
\hline \multicolumn{2}{|c|}{ Shear modulus $G$} & & \multicolumn{2}{|l|}{103.0} & \multicolumn{2}{|l|}{130.8} & \multicolumn{2}{|l|}{51.4} & \\
\hline \multicolumn{2}{|c|}{ Young's modulus $E$} & & \multicolumn{2}{|l|}{258.4} & \multicolumn{2}{|l|}{332.9} & \multicolumn{2}{|l|}{115.3} & \\
\hline \multicolumn{2}{|c|}{ Poisson's ratio $\eta$} & & \multicolumn{2}{|l|}{0.255} & \multicolumn{2}{|l|}{0.273} & \multicolumn{2}{|l|}{0.123} & \\
\hline
\end{tabular}

${ }^{\mathrm{a}}$ Reference 60.

attempted except for the basal model mentioned earlier. Experimentally, the mechanical properties of silicon nitride and the effect of impurity-doped microstructures such as IGFs have been well investigated. ${ }^{15,39-42}$ It was concluded that the evolution of microstructures in $\beta-\mathrm{Si}_{3} \mathrm{~N}_{4}$ is greatly affected by the adsorption of rare-earth elements at the grain surfaces and by the change in viscosity of the intergranular phases. However, the atomistic scale connection between the mechanical properties and the electronic bonding in the IGF structure has not been studied in detail. Here we present the mechanical and elastic properties of the prismatic model using $a b$ initio methods.

The mechanical and elastic properties of the prismaticIGF model are calculated using the stress vs strain approach. ${ }^{56}$ A small strain of $-1 \%$ (compression) and $+1 \%$ (expansion) are applied to each independent strain element of the IGF model. The slightly deformed structure is then fully optimized while keeping the volume constant. The six components of the stress data $\left(\sigma_{i}\right)(x x, y y, z z, y z, z x, x y)$ under applied strains $\left(\varepsilon_{i}\right)$ are collected and the elastic tensor $C_{i j}$ is obtained by solving the resulting set of linear equations. Using the Voigt-Reuss-Hill averaging approximation appropriate for polycrystalline samples,${ }^{57}$ the bulk modulus $(K)$, shear modulus $(G)$, Young's modulus $(E)$, and Poisson's ratio $(\eta)$ were evaluated. These numbers are obviously very meaningful for the IGF model studies since $\beta-\mathrm{Si}_{3} \mathrm{~N}_{4}$ is a polycrystal with crystalline grains riddled with IGF type microstructures. The same approach has been used by us on several crystalline systems ${ }^{58,59}$ with results close to the experimental data. In the present case for the IGF model, the calculation is obviously computationally far more demanding due to the system size.

The calculated elastic constants and the resulting mechanical properties in the form of the bulk modulus $K$, shear modulus $G$, Young's modulus $E$, and Poisson's ratio $\eta$ for the prismatic-IGF model are listed in Table II. For comparison, we have also calculated the elastic constants and the same mechanical properties parameters for hexagonal crystalline $\beta$ - $\mathrm{Si}_{3} \mathrm{~N}_{4}$ and compared them with the elastic constants from single-crystal measurements of $\beta-\mathrm{Si}_{3} \mathrm{~N}_{4} \cdot{ }^{60}$ The agreement between the two is excellent. As can be expected, the elastic constants of the IGF model are all smaller than the crystalline counterpart indicating the weakened interatomic bonding in the glassy region. In this comparison, it should be noted that the IGF model is in the form of an orthorhombic cell with the $z$ axis in the direction perpendicular to the IGF layer whereas in the hexagonal crystalline $\beta-\mathrm{Si}_{3} \mathrm{~N}_{4}$ the $z$ axis coincides with the [001] direction. The hexagonal crystalline $z$ axis corresponds with the IGF model's $x$ axis. If we assume that the mechanical properties of the prismatic-IGF model are proportional to the volume fractions of the crystalline portion and the glass portion, as would be expected from macroscale samples in continuum mechanics, we can estimate the elastic tensor for the IGF portion of the model and use it to calculate the values for $K, G, E$, and $\eta$. They are listed in Table II. It can be seen that the calculated bulk and Young's moduli for the glassy film are 51.0 GPa and 115.3 $\mathrm{GPa}$, respectively. This value is similar to the reported bulk modulus values from a simulation study of silicon oxynitride glass using a continuous random network model ${ }^{61}(K$ $=50-90 \mathrm{GPa}$ for weight percentage of $\mathrm{Si}_{3} \mathrm{~N}_{4}$ from $20 \%$ to $60 \%$ ).

In addition to the mechanical properties, we have also calculated the longitudinal component of sound velocity for the prismatic-IGF model from the elastic data in the three orthogonal planes by solving the Christoffel wave equation, ${ }^{62}$

$$
\rho v^{2} u_{i}=C_{i j k l} l_{j} l_{k} u_{l},
$$

where $\rho$ is the density, $v$ is the elastic wave velocity, $C$ is the elastic tensor, $\ell$ is the direction of the $k$ vector, and $u_{i}$ is the polarization of the elastic wave. The results are displayed in Fig. 2 for the three orthogonal planes of the model. In Fig. 2, the two inner curves are the transverse components and the large outer one is the longitudinal sound velocity. On the first instinct, one would expect that the presence of a glassy layer with a composition different from the crystalline part would 

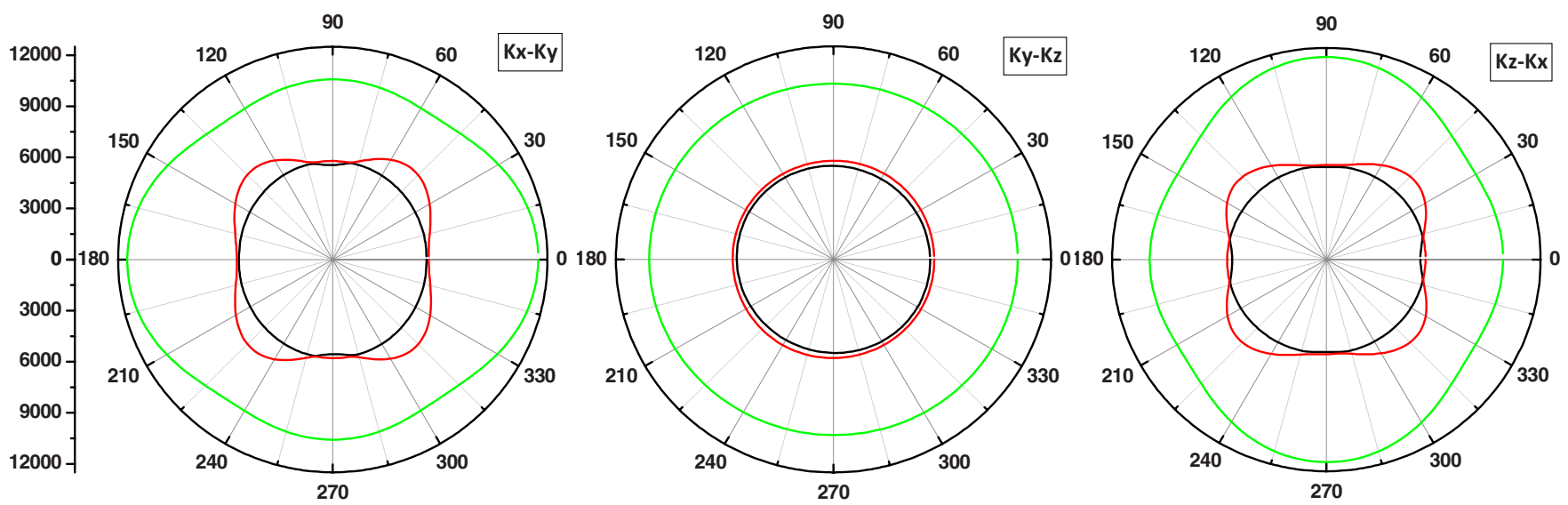

FIG. 2. (Color online) The calculated longitudinal elastic sound velocity profile of the IGF model: (a) $k_{x}-k_{z}$ plane; (b) $k_{x}-k_{y}$ plane; and (c) $k_{z}-k_{y}$ plane. Green: longitudinal; red and blue: transverse. The result shows that the sound velocity (in units of meter per second) is not affected by the presence of the IGF but depends on the orientation of the crystalline planes.

certainly affect the longitudinal sound velocity in this direction (the $z$ direction). The result in Fig. 2 shows that this is actually not the case. The longitudinal component of the sound velocity in the $\mathrm{Y}-\mathrm{Z}$ plane is almost isotropic ranging from 10300 to $10580 \mathrm{~m} / \mathrm{s}$ whereas in the X-Y and Z-X planes, the variation is quite large. Furthermore, the sound velocity distribution in $\mathrm{X}-\mathrm{Y}$ and $\mathrm{Z}-\mathrm{X}$ planes resemble each other if rotated by $90^{\circ}$. In the $\mathrm{X}-\mathrm{Y}$ plane, the maximum velocity of $11957 \mathrm{~m} / \mathrm{s}$ is along the $x$ direction and the minimum velocity of about $10220 \mathrm{~m} / \mathrm{s}$ is $60^{\circ}$ off the $x$ axis. In the $\mathrm{Z}-\mathrm{X}$ plane, the maximum velocity of $11958 \mathrm{~m} / \mathrm{s}$ is along the $x$ direction and the minimum velocity of $10059 \mathrm{~m} / \mathrm{s}$ is about $30^{\circ}$ off the $z$ axis. The fact that the sound velocity in the $x$ direction is different than that in the other directions is consistent with the structure of $\beta-\mathrm{Si}_{3} \mathrm{~N}_{4}$, and the probable reason is the columnar arrangement of atoms along the crystalline $c$ axis compared to any orientation in the crystalline $a-b$ plane. The sound velocity in polycrystalline $\mathrm{Si}_{3} \mathrm{~N}_{4}$ is not affected by the presence of IGFs but depends most strongly on the bulk crystalline orientation. We will return to this point in Sec. VI for more detailed discussion.

\section{B. Electronic structure and bonding}

The electronic structure and bonding is the fundamental property of a solid on which many other physical properties rely. This is particularly important for the mechanical properties described in the previous section. For IGF models in polycrystalline ceramics, the basic questions are how the electronic structure is affected by the presence of the glassy layer and how the interatomic bonding in the glassy region and at the glass/crystalline interface are different from the bonding in the bulk crystal. These questions can only be answered by rigorous quantum-mechanical calculations on a realistic model and cannot be inferred from classical treatments of density, composition, and atomic arrangement alone.

The electronic properties of the prismatic-IGF model are calculated using the OLCAO method described in Sec. II.
Figure 3 shows the calculated density of states (DOS) of the IGF model including a comparison with the total DOS of crystalline $\beta-\mathrm{Si}_{3} \mathrm{~N}_{4}$. The total DOS can be resolved into different types of atoms or orbital components. It can also be resolved into different regions of the model (crystalline or IGF). In Fig. 3, the zero of the energy is set at the highest occupied state which could be a defect-induced state in the case of the IGF model and may not be at the top of the valence band (VB) except in the case of bulk crystalline $\beta-\mathrm{Si}_{3} \mathrm{~N}_{4}$ [Fig. 3(a) second panel]. The occupied VB for the IGF model consists of two segments. The lower one ranges from -15 to $-22.5 \mathrm{eV}$ and consists mainly of $\mathrm{O} 2 s, \mathrm{~N} 2 s$, and $\mathrm{Si} 3 s$ orbitals while the upper $12.5 \mathrm{eV}$ wide segment consists of $\mathrm{O}$ and $\mathrm{N} 2 p$ orbitals, and $\mathrm{Si} 3 p$ orbitals (with some Si $3 d$ mixing). The main difference in the DOS between the IGF model and $\beta-\mathrm{Si}_{3} \mathrm{~N}_{4}$ is that the IGF model has a larger band width, for both the lower and upper segments, due to the presence of $\mathrm{O}$ ions in the IGF region which have a lower binding energy than $\mathrm{N}$.

An interesting observation is that the band gap of the IGF model (excluding the defect-induced states) is comparable to bulk $\beta-\mathrm{Si}_{3} \mathrm{~N}_{4}$, (close to $4.0 \mathrm{eV}$ ). This gap value is of course underestimated due to the LDA approximation in DFT. There exist one unoccupied and seven occupied defect states in the gap above the VB edge and three defect states below the bottom of the conduction-band edge. These defect states can be traced to a few undercoordinated or overcoordinated atoms discussed in Sec. III. To illustrate this further, we have calculated the localization index (LI) of all the electron states (labeled $n$ ) in the IGF model according to

$$
\mathrm{LI}_{n}=\sum_{i, \alpha}\left[\sum_{i, \beta} C_{i \alpha}^{* n} C_{j \beta}^{n} S_{i \alpha, j \beta}\right]^{2}
$$

They are plotted in Fig. 4. It shows that the defect states near the top of the VB are highly localized with LI larger than those of the localized states at the VB edge. In a pure amorphous glass represented by an ideal continuous random 

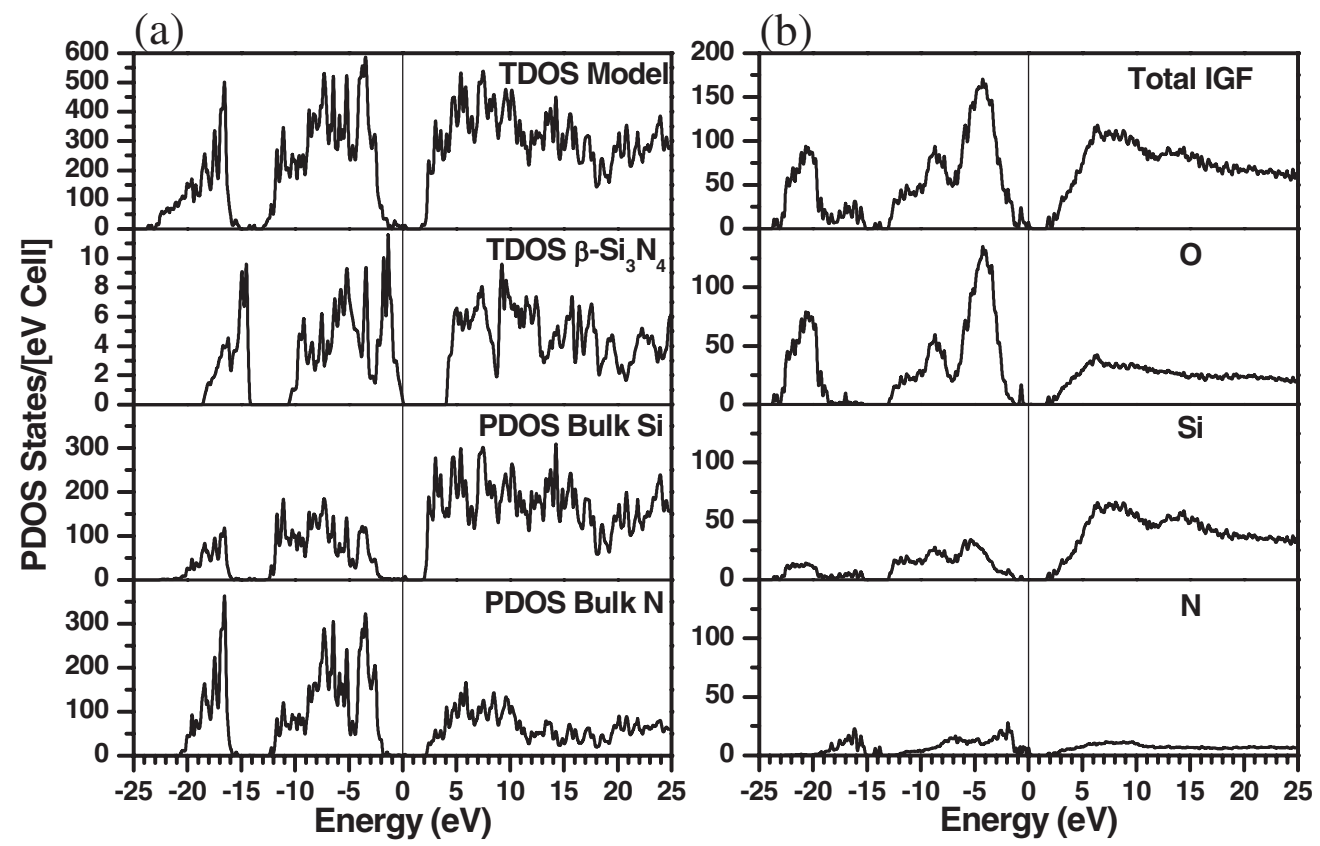

FIG. 3. Calculated total and PDOS of the IGF model: (a) DOS of bulk $\mathrm{Si}_{3} \mathrm{~N}_{4}$ for the IGF model and crystalline $\beta$-Si ${ }_{3} \mathrm{~N}_{4}$ (top 2 panels) and the $\mathrm{Si}$ and $\mathrm{N}$ components in the bulk region of the IGF model (lower 2 panels); (b) DOS of the atoms in the glassy region of the IGF model and its atomic components. The zero of the energy is set at the highest occupied level which could be a defect level and is not necessarily the top of the VB except in the case of crystalline $\beta-\mathrm{Si}_{3} \mathrm{~N}_{4}$.

network model, ${ }^{63}$ there are no defective states but states at the band edges (both the $2 s$ band and the upper VB) are relatively localized in accordance with Anderson's localization theory. ${ }^{64}$ The $\alpha-\mathrm{SiO}_{2}$ model, though noncrystalline, has a near-perfect perfect short-range order but no broken bonds and therefore no defective states. ${ }^{63}$ The absolute value of the localization index value depends on the size of the model. The presence of the IGF in $\beta-\mathrm{Si}_{3} \mathrm{~N}_{4}$ definitely results in defectlike states which could act as potential traps not present in a perfect glass and could have larger implications on using $\mathrm{Si}_{3} \mathrm{~N}_{4}$ in microelectronic devices.

The effective charge $Q_{\alpha}^{*}$ for every atom in the IGF model and the BO values for every bonded pair of atoms in the IGF model are calculated according to Eqs. (1) and (2) and displayed in Fig. 5. Figure 5(a) shows the $Q^{*}$ for $\mathrm{Si}, \mathrm{N}$, and $\mathrm{O}$ atoms across the $z$ axis. The averaged effective charge for $\mathrm{Si}$ in the bulk crystalline region is 2.41 valence electrons and

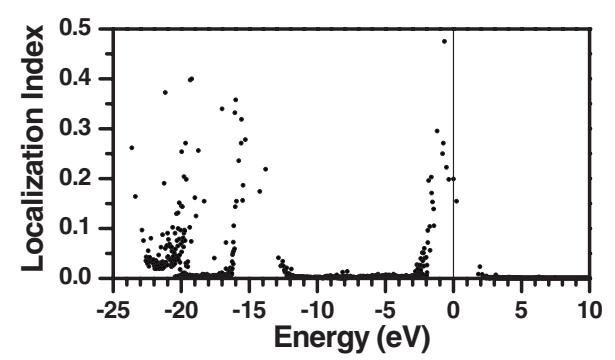

FIG. 4. Calculated LI of the prismatic-IGF model across the entire energy range. Each dot represents the LI of one state. The defect-induced states near the gap are highly localized. that in the IGF region is 2.02 valence electrons. For N, the averaged $Q^{*}$ is 6.19 valence electrons in the bulk region and 6.18 valence electrons in the IGF region. The $\mathrm{O}$ ions are present only in the IGF region and have an averaged $Q^{*}$ of 7.03 valence electrons. These numbers indicate that the IGF is more ionic than the crystalline part which was anticipated. It is also noted from Fig. 5(a) that the variations in $Q^{*}$ within the IGF can be fairly large with some anions actually losing charge and becoming nominally cationic. This is of course related to the disordered and defective nature of the atoms within IGF and their interactions which can only be revealed the $a b$ initio quantum-mechanical calculations.

The calculated BO values for all atomic pairs are displayed in Fig. 5(b). The same data plotted as colored sticks for the bonds are shown in Fig. 5(c). Within the IGF, very strong and very weak bonds exist depending of the bond length and to a lesser extent, the bond angles. The Si-O bonds are generally weaker than $\mathrm{Si}-\mathrm{N}$ bonds. As will be shown later, the fracture of the IGF under tensile strain starts at the location within the IGF that has a concentration of weak bonds. In the crystalline portion of the IGF model, the $\mathrm{BO}$ values are constant as expected [the $\beta-\mathrm{Si}_{3} \mathrm{~N}_{4}$ crystal has 2 (3) different types of $\mathrm{Si}-\mathrm{N}$ bond lengths and hence slightly different $\mathrm{BO}$ values ${ }^{65}$ ]. However, it is also observed that at the glass/crystalline interface, the variations in $\mathrm{BO}$ start to increase and tend to penetrate at least several crystalline planes into the bulk region. This indicates that as far as bond orders (reflecting the bond lengths) are concerned, the interphase boundary is not abrupt even though the integrity of the $\mathrm{Si}-\mathrm{N}$ bonds remain and there are no $\mathrm{O}$ ions diffusing into the bulk layers as was the case for the basal model..$^{35}$ 

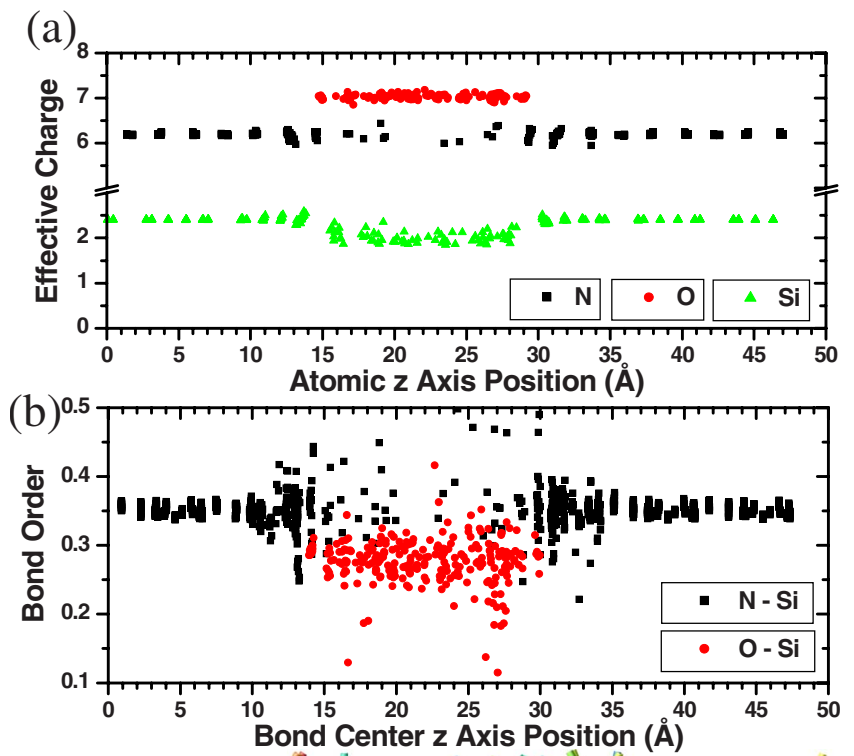

(c)
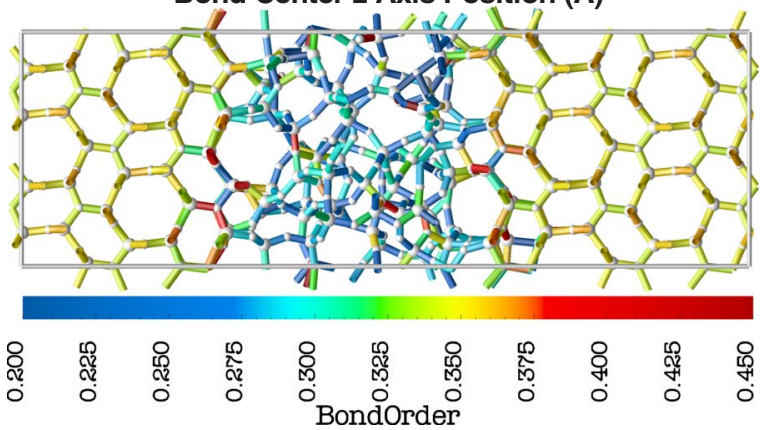

FIG. 5. (Color online) (a) Calculated Mulliken effective charges $Q^{*}$ for $\mathrm{Si}, \mathrm{N}$, and $\mathrm{O}$ along the $z$ axis. (b) Calculated bond order values along the $z$ axis and averaged over the $x-y$ plane. (c) Same as (b) but illustrated in a color scheme with stronger bonds in dark red and weaker bonds in dark blue.

\section{Electrostatic potential difference}

The space-charge model in ceramic materials has been a well established concept supported by ample experimental evidence. ${ }^{6-71}$ It originates from the presence of ionic charges that can result in an electrostatic potential difference and which will significantly affect the electric conductivity of the ionic crystals and plays a role in the balance of force model for the IGF formation in ceramics. ${ }^{10}$ Thus, the spacecharge model generally implies that charges will accumulate at the grain boundaries or at the interfaces of microstructures such as IGFs that result in a difference in electrostatic potential across the GBs or IGFs that can affect charge transport and other related properties. The space-charge model has been established mainly from indirect experimental observations and, to our knowledge, has not been adequately addressed or demonstrated by rigorous theoretical calculations. Part of the reason is related to the length scale of the phenomenon. The space-charge model generally covers a length scale of a sample in the micrometer range whereas the largest models for grain boundaries or IGFs with which accurate $a b$ initio calculations can be attempted are probably much less than $10 \mathrm{~nm}$. In our previous study of the basal model, ${ }^{36}$ it was demonstrated that there is a difference in the average

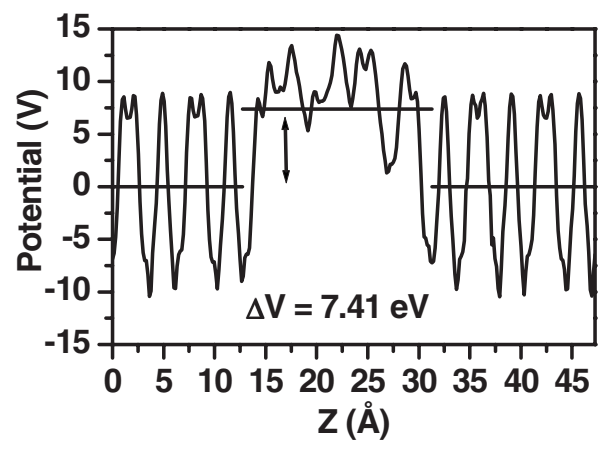

FIG. 6. Calculated electrostatic potential from self-consistent DFT-LDA of the IGF model along the $z$ axis and averaged over the $x-y$ plane. The zero potential is set at the averaged potential in the bulk region. The horizontal line indicates the averaged value in the IGF region.

electrostatic potentials within the IGF region and the bulk crystalline region on the order of $2.58 \mathrm{eV}$. This difference is increased when the IGF region is doped with $\mathrm{Y}$ ions. Although the size of the basal model was relatively small (29.575 $\AA \times 22.845 \AA \times 13.119 \AA$ with 798 atoms), the evidence is clear that there is an electrostatic potential difference due to the presence of an IGF in support of the spacecharge model. The fact that there exists a potential difference between a microstructure is of course predicted by the spacecharge model and used in the interpretation of many experimental observations but it is seldom discussed or demonstrated in actual simulations. The validity of the space-charge model also has implications on the equilibrium forces in the formation of IGFs (it can be either attractive or repulsive depending on the sign of the charges). ${ }^{10,11}$ Calculation of the electrostatic potential across an IGF provides some estimation for the nature and strength of the force originating from the space-charge model.

Figure 6 shows the calculated electrostatic potential in the prismatic-IGF model along the $z$ axis and averaged over the $x-y$ plane. They are obtained from the final self-consistent potential in the OLCAO calculation and evaluated on a dense $50 \times 50 \times 300$ mesh. In the bulk crystal region, the potential curve shows regularly spaced deep valleys and sharp peaks as the $x-y$ plane passes through atomic layers where $\mathrm{Si}$ or $\mathrm{N}$ atoms lie. In the IGF region, the structure is amorphous so the curve displays no regularities due to the inhomogeneity of the ion distribution within the IGF. It is obvious that the averaged electrostatic potential in the IGF region (dashed line in Fig. 6) is higher than the electrostatic potential in the crystalline region by as much as $7.41 \mathrm{eV}$. The lines are drawn and the potential difference is taken by using the average values for the IGF range including the first trough as opposed to the last peak. These are of course approximate values with a certain range of uncertainty since the boundary between the crystal and IGF is difficult to define. Thus the potential difference between the IGF and bulk crystal depends on the crystalline orientation of the IGF and also on the IGF's chemical composition.

\section{Optical dielectric functions}

The optical dielectric function of the prismatic-IGF model is calculated according the following expression for crystal- 


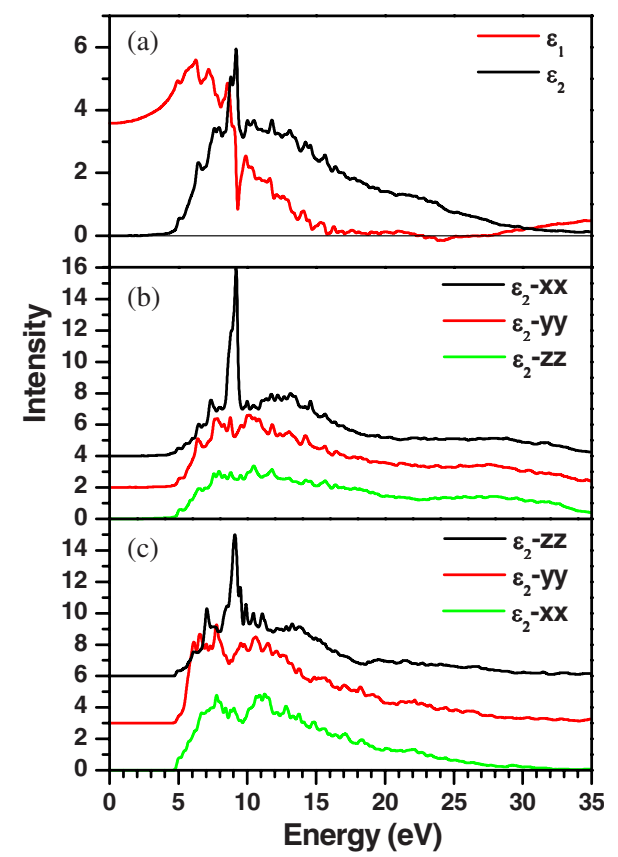

FIG. 7. (Color online) (a) Calculated optical dielectric function of the IGF model; real part (red) and imaginary part (black). (b) Imaginary dielectric function of the IGF model resolved in the three directions $(x, y, z)$.(c) Imaginary dielectric function of crystalline $\beta-\mathrm{Si}_{3} \mathrm{~N}_{4}$ resolved in the three directions $(x, y, z)$.

line solids using the wave functions obtained from the OLCAO electronic structure calculation:

$$
\begin{aligned}
\varepsilon_{2}(\hbar \omega)= & \frac{e^{2}}{\pi m \omega^{2}} \int_{\mathrm{BZ}} d k^{3} \sum_{n, l}\left|\left\langle\psi_{n}(\vec{k}, \vec{r})|-i \hbar \vec{\nabla}| \psi_{l}(\vec{k}, \vec{r})\right\rangle\right|^{2} \delta\left[E_{n}(\vec{k})\right. \\
& \left.-E_{l}(\vec{k})-\hbar \omega\right] .
\end{aligned}
$$

Because the model has a large volume, the use of only one $k$ point at $(0,0,0)$ for Brillioun zone integration in Eq. (5) is sufficient. Figure 7(a) shows the real and imaginary parts of the dielectric function up to $35 \mathrm{eV}$. The real part $\varepsilon_{1}(\hbar \omega)$ is obtained from the imaginary part $\varepsilon_{2}(\hbar \omega)$ using KramersKronig conversion. Figure 7(a) shows that for the IGF model, $\varepsilon_{2}(\hbar \omega)$ has a sharp absorption peak at $9.0 \mathrm{eV}$ and $\varepsilon_{1}(\hbar \omega)$ has a zero-frequency limit of 3.57 implying a refractive index of about 1.89. Figure 7(b) shows the three components of the $\varepsilon_{2}(\hbar \omega)$ curve. It is clear that the sharp peak at $9.0 \mathrm{eV}$ comes exclusively from the $x$ component so the strong anisotropy in $\varepsilon_{2}(\hbar \omega)$ in the IGF model is not due to the presence of the IGF with its glassy layer perpendicular to the $z$ axis but is related to crystal orientation of the bulk $\beta-\mathrm{Si}_{3} \mathrm{~N}_{4}$. This conclusion is confirmed by the optical results for crystalline $\beta-\mathrm{Si}_{3} \mathrm{~N}_{4}$ in Fig. 7(c) showing the same anisotropic pattern. This effect is similar to the case of longitudinal sound velocity shown earlier in Sec. IV A.

\section{THEORETICAL TENSILE EXPERIMENT}

Many intriguing mechanical properties of materials containing microstructures such as fracture toughness, plasticity, and creep resistance are difficult to measure in laboratory

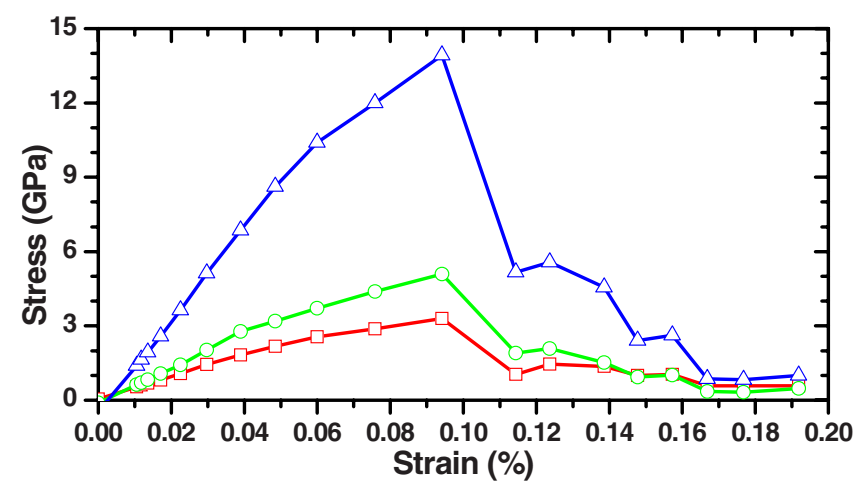

FIG. 8. (Color online) Strain vs stress curve of the IGF model under tensile extension in the $z$ direction.

experiments due to problems related to sample characterization and a lack of available theoretical models for their proper interpretation. Mesomechanical models assume that silicon nitride grains are rigid during the deformation and that the displacement between adjacent grains occurs only at the GB or IGF. Large-scale computational modeling at the atomic level can fulfill the role of developing the much needed theoretical models that may lead to additional understanding on topics such as intergranular or transgranular failure behavior, cavitation model, ${ }^{72}$ tensile creep ${ }^{73}$ etc.

\section{A. Method of simulation}

We have carried out a theoretical tensile experiment on the prismatic-IGF model using a massively parallel supercomputer at the National Energy Research Supercomputing Center (NERSC). Some preliminary results were reported earlier. ${ }^{44}$ Similar tensile experiments have also been carried out with the basal mode ${ }^{36}$ and on a $\Sigma=3$ grain-boundary model in $\alpha-\mathrm{Al}_{2} \mathrm{O}_{3}$ at a much smaller scale. ${ }^{74}$ Here we present a more complete set of better converged data from the theoretical tensile experiment. The simulation was carried out by systematically extending the initial model (Fig. 1), which is calibrated to be at zero strain in the $z$ direction, in small steps with the $x-y$ dimensions kept fixed. At each step of incremental elongation of the $z$ axis, the model is fully relaxed using VASP until the desired convergence is reached. The relaxed structure is then used as the starting structure for the next strain level and fully relaxed again. The cycles were repeated until the model was fully fractured and well separated. The total energy, stress components, and atomic configuration of the extended IGF model at each step were registered. A total of 20 data points were collected. A significant amount of computing resources was committed to obtain these data. A subset of the strained configurations was selected for electronic structure and bonding calculations using the OLCAO method as described in the previous section.

\section{B. Data analysis from theoretical tensile experiment}

Figure 8 shows the calculated stress $\sigma$ vs strain s for the three Cartesian components $\sigma_{z z}, \sigma_{x x}$, and $\sigma_{y y}$ from the theoretical tensile experiment. The strain $s$ is defined as the percentage of extension of the entire simulation cell in the $z$ 


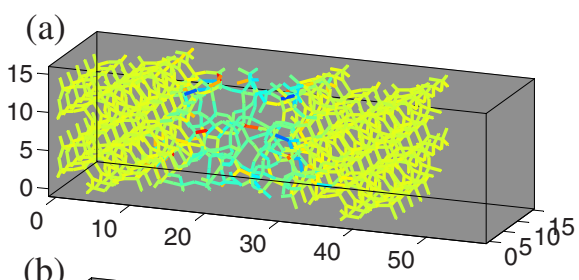

(b)
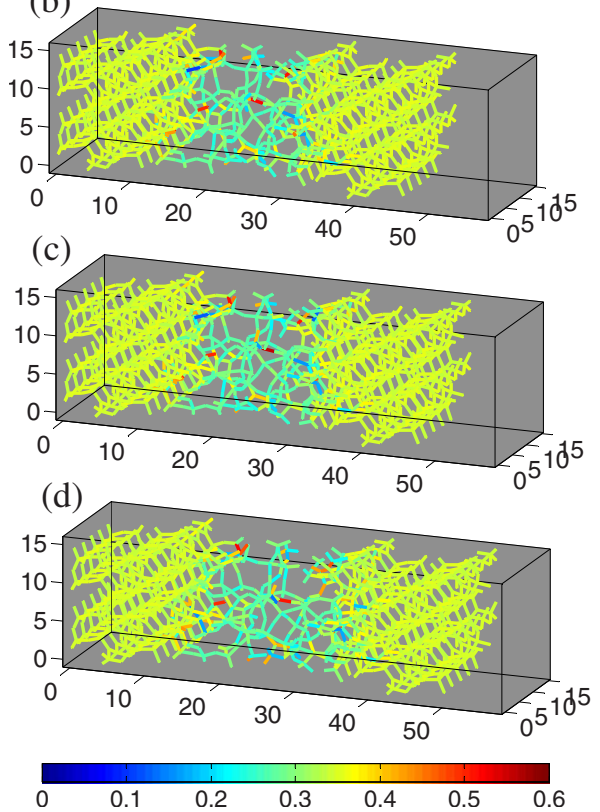

FIG. 9. (Color online) Evolution of the BO values of the IGF model under tensile strains. (a) $s=0$; (b) $s=1.7 \%$; (c) $s=3.9 \%$; and (d) $s=9.4 \%$. There are no abrupt changes in the $\mathrm{BO}$ values at these strains but the presence of both very strong bonds and weak bonds reflected by $\mathrm{BO}$ values remains.

direction. In this experiment, we have fixed the dimension of the cell in the $x$ and $y$ directions. This corresponds to a "uniaxial extension" and it will induce a triaxial stress state. ${ }^{75}$ As expected, the $z z$ component is much larger than the $x x$ and $y y$ components since the load is applied in the $z$ direction. Figure 8 shows some interesting features in both the prefailure and postfailure regions. The stress vs strain relation is almost linear up to $s=0.05$ and then it changes its slope until it reaches a maximum stress of $13.9 \mathrm{GPa}$ at the strain of $s=0.094$. In contrast, the peak stress for perfect $\beta-\mathrm{Si}_{3} \mathrm{~N}_{4}$ crystals has been reported to be as high as $75 \mathrm{GPa}$ at strains greater than $25 \% .^{76}$ For $\beta$-SiAlON $\left(\mathrm{Si}_{6-z} \mathrm{Al}_{z} \mathrm{O}_{z} \mathrm{~N}_{8-z}\right)$, the peak stress (strain) ranges from $27 \mathrm{GPa}(13 \%)$ to $49 \mathrm{GPa}$ (17\%) for $z$ ranging from 1 to $5 .{ }^{77}$ Unlike known brittle materials, $\sigma_{z z}$ for the prismatic-IGF model does not drop to zero after reaching the maximum stress. Even at a strain of 0.124 , where the IGF model begins to fracture, the stress is $5.57 \mathrm{GPa}$, or $40 \%$ of the maximum value. At $s=0.157$ where the IGF has already fractured, there remains a sizable residual stress of $2.62 \mathrm{GPa}$ or $19 \%$ of the maximum value. Thus the IGF model shows some elements of plastic behavior under tensile strain.

The variation in $\mathrm{BO}$ values for the IGF model under strain are presented in Fig. 9 as a sequence of three-dimensional color plots for strain levels of $s=0.0,0.017,0.039$, and 0.094. The BO values depend mostly on the bond lengths but they can also be affected by the presence of other atoms or variations in bond angle. The IGF model's fracture resistance under tensile strain depends to a large extent on the number of bonds present and the strength of those bonds. It is clear that even at zero strain, there are very strong and very week $\mathrm{Si}-\mathrm{O}$ and S-N bonds as already discussed in Sec. IV B. At small strain, the bonds within the IGF were simply elongated. At a larger strain bonds can start to break and $\mathrm{BO}$ values will change as a result. ${ }^{44}$ At the largest strain simulated $(s=0.192)$, the two parts of the model were clearly separated and the model resembles two $\beta-\mathrm{Si}_{3} \mathrm{~N}_{4}$ (001) surfaces coated with glassy films. (The postfailure behavior of the IGF model will be discussed below.) The fracture of the model under increased strain starts at the interior portion of the IGF with lower bond density [see Fig. 9(d)], not at the interface between the IGF and the crystal. This is very different from the basal IGF model where the fracture was found to occur at the IGF crystal interface and the stress dropped to zero immediately after fracture. ${ }^{36}$

Figure 10 shows the components of the strain field distribution $\left(\varepsilon_{z z}, \varepsilon_{y y}\right.$, and $\left.\varepsilon_{x x}\right)$ of the IGF model for strains of $s$ $=0.135,0.225,0.390$, and 0.942 . Here we use the notation $\varepsilon_{z z}$ to denote the components of the local strain field in accordance with the general practice in engineering mechanics and it should not be confused with the dielectric function discussed in Sec. IV D. The strain field is obtained as the displacement pattern of atoms from the equilibrium configuration $(s=0)$ using a practical scheme we developed following ideas from continuum mechanics. ${ }^{38}$ In this scheme the model cell is divided into $\mathrm{n}$ grid points. A linear displacement field associated with the $n$th grid point is calculated such that the displacement of the $p$ th atom within a small group of local atoms in a sphere of radius $R_{o}$ centered at the $n$th grid point is expressed as the displacement gradient $d_{i j}$, and written in terms of the local deformation gradient $F_{i j}$. The local Green-Lagrange strain tensor $\varepsilon_{i j}^{n}$ is then obtained from $F_{i j}^{n}$. The local strain field describes the collective stiffness behavior of a few atoms at various locations within the atomistic model. The calculated numerical values for the strain field components are plotted in color on a dense mesh of points for visualization. Even at a small strain of 0.0135, $\varepsilon_{z z}$ in the IGF is highly visible whereas $\varepsilon_{y y}$ and $\varepsilon_{x x}$ are negligible. As the strain is increased, $\varepsilon_{z z}$ becomes more intensified. At the strain of 0.0942 [Fig. 10(d)] the strain is transmitted to the crystalline portion and both $\varepsilon_{y y}$ and $\varepsilon_{x x}$ become visible in the IGF region.

It is also interesting to see how some of the physical properties change under the $z$ axis uniaxial strain. The DOS under strain does not change very much at all. ${ }^{44}$ Figure 11 shows the electrostatic potential distribution under increasing strain including the zero strain case already shown in Fig. 6 up to a strain of 0.094 where the stress reaches a maximum. The difference in the averaged electrostatic potential between the IGF region and the bulk crystalline region steadily increases as the strain is increased [Fig. 11(b)]. This is consistent with the fact that the increased strain basically increases the volume of the IGF region with an accompanying reduction in charge per unit volume whereas the volume of the bulk crystal portion hardly changes at all. Such analysis of the electrostatic potential difference could be important in micro- 
(a)

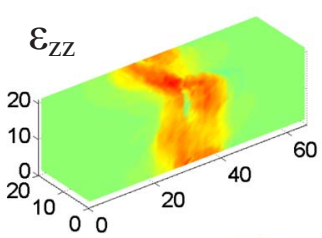

(b)

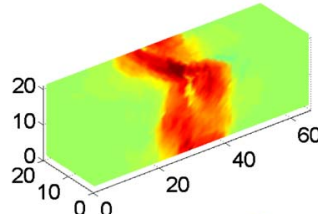

(c)

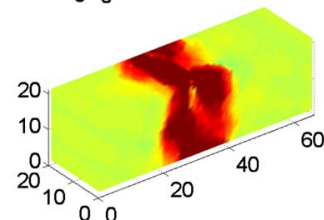

(d)

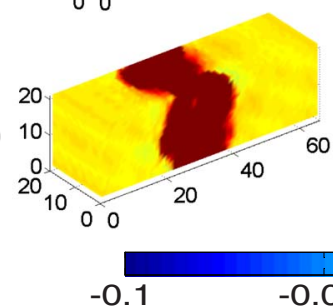

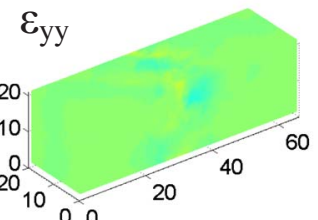
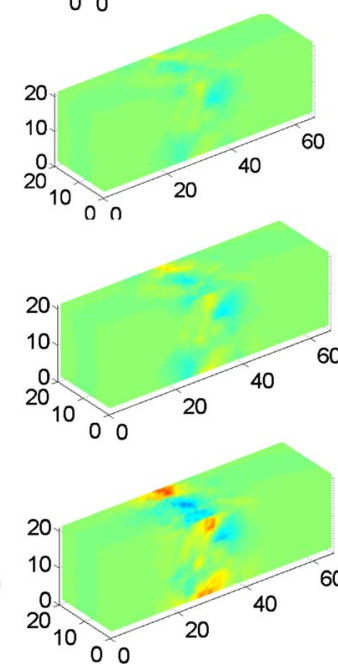
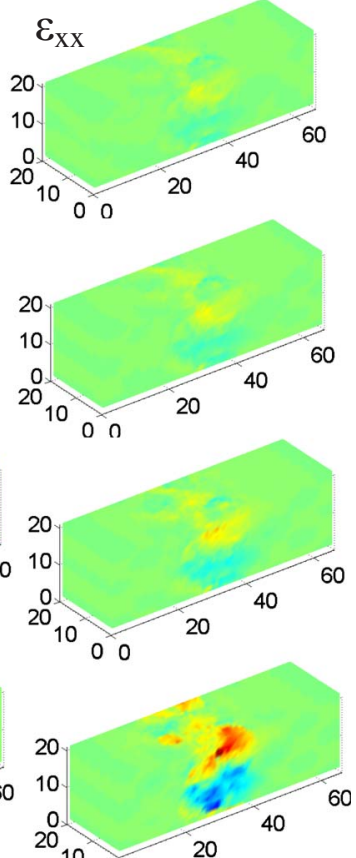

FIG. 10. (Color online) Evolution of the strain field plots in three Cartesian directions $\left(\varepsilon_{x x}\right.$, $\varepsilon_{y y}$, and $\varepsilon_{z z}$ ) with reference to the unstrained model $(s=0)$. (a) $s$ $=1.35 \%$; (b) $s=2.25 \%$; (c) $s$ $=3.90 \%$; and (d) $s=9.42 \%$. (a)
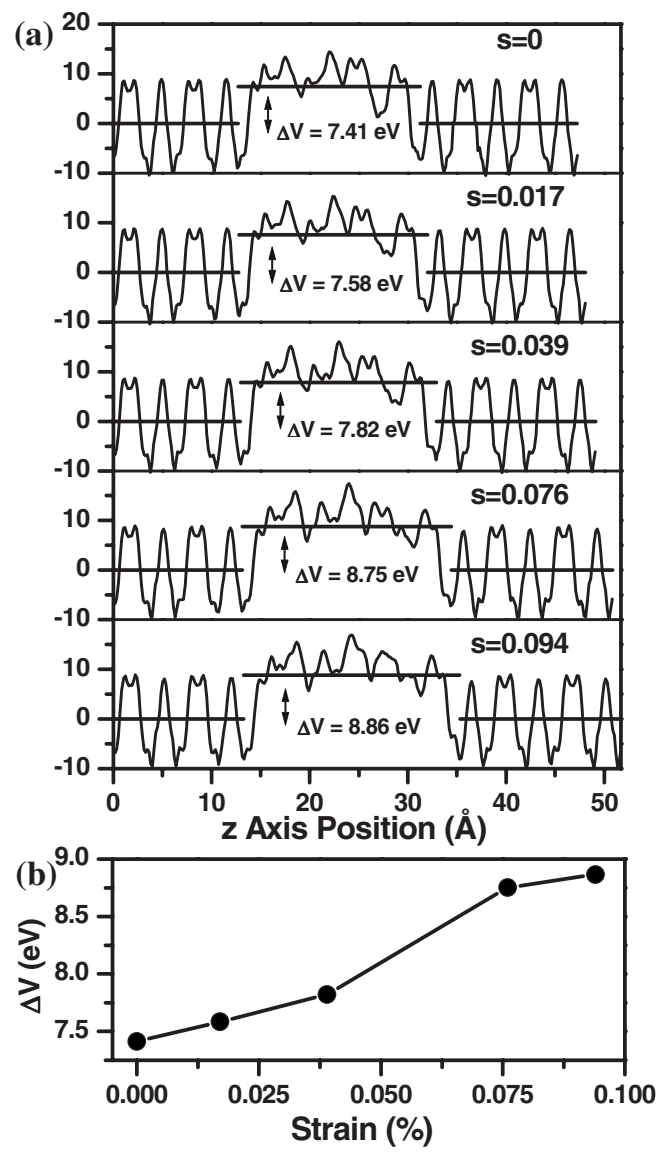

FIG. 11. (a) Change in the electrostatic potential across the IGF as a function of strain up to $s=0.094$. The notations are the same as in Fig. 6. (b) Plot of $\Delta V$ vs strain for five data points in (a). electronics where the device performance may depend on the strain induced by the interface.

\section{Postfailure analysis}

As the strain is further increased the IGF eventually fractures. Figure 12(a) shows the ball and stick model of the fractured IGF at $s=0.192$. The model evolved into two surfacial films on the crystalline surfaces of the bulk $\beta-\mathrm{Si}_{3} \mathrm{~N}_{4}$ crystal separated by a vacuum region. As already pointed out earlier, even at this strain the stress is not zero. The residual stress on the surfacial films is the result of strains associated with the readjustment and recoil of surface bonds. Figure 12(b) shows the calculated DOS of this fractured model. A surprising fact is that the overall DOS features change very little compared to the intact model. The "gap" remains about the same size as in the original unstrained model except that more localized states appear near the VB edge. These are surfaces states from the surfacial films in addition to the defective states that remain.

Figure 12(c) shows the electrostatic potential distribution of the fractured model. The center of the plot is at the vacuum region of the fractured IGF model. The electrostatic potential difference between the vacuum and the crystal part of the model is estimated to be about $24 \mathrm{eV}$. The difference between the surfacial films and the bulk $\beta-\mathrm{Si}_{3} \mathrm{~N}_{4}$ is about 8 $\mathrm{eV}$. Such values need to be treated with caution and they are useful primarily for qualitative estimations for the reason already elaborated on in Sec. IV C. Figure 12(d) shows the calculated optical dielectric function of the fractured model with a vacuum region. Compared to the zero strain model of Fig. 7, the overall changes in spectral features are quite small. The sharp peak remains at about $9 \mathrm{eV}$ and the optical 

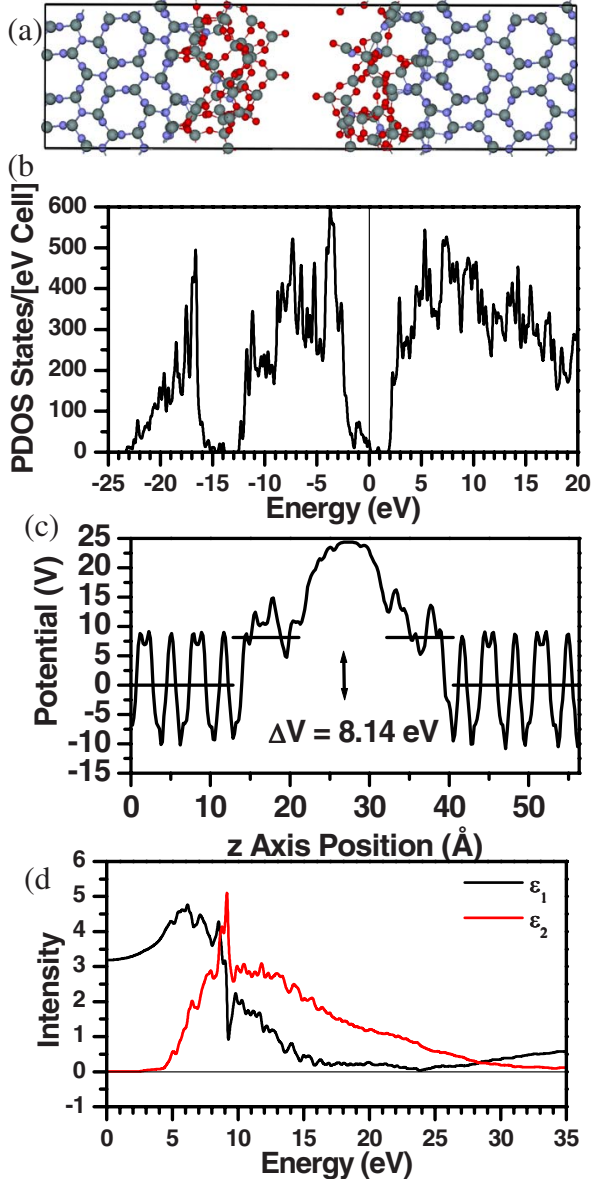

FIG. 12. (Color online) Analysis of the postfailure behavior of the prismatic-IGF model: (a) Ball and stick model; (b) total DOS; and (c) electrostatic potential across the fractured IGF model. (d) Real and imaginary parts of the dielectric function.

dielectric constant $\varepsilon_{1}(0)$ is now reduced to 3.2 due to the presence of the vacuum region.

\section{DISCUSSION}

The results of $a b$ initio simulations presented in the previous two sections provide a wealth of information on several different but interconnected physical properties of a realistic IGF model and its response under tensile strain. Such data are not easy to obtain directly from experiment and provide us some incisive insights on atomic level behavior in such complex microstructures. For the IGF model under zero strain, we find the mechanical properties and elastic deformation behavior to be very different from the basal model, underscoring the importance of crystalline orientation relative to the glassy region and the role of defective structures at the crystal/glass interface. In the basal model, the stressstrain relationship does not follow the Cauchy-Born rule. In the prismatic-IGF model, the initial strain/stress relationship is linear. It then changes slope at $s=0.05$ and reaches a maximum stress of $13.9 \mathrm{GPa}$ at $s=0.094$. This prismatic-IGF model with a precisely defined IGF thickness and exact composition enables us to explore the role of $\mathrm{N}$ ions in the glassy layer which is known to increase the viscosity of the IGF and thus affect its mechanical properties and plastic behavior. We are able to relate the mechanical and elastic properties of the IGF model to the fundamental electronic structure and bonding through detailed bond order and effective charge calculations. The quantitative parameters obtained in this study can eventually be connected to parameters needed for multiscale simulations at a larger length scale. This is an achievable, direct, and accurate way to build a bridge between fundamental physics and materials engineering.

The results of the electronic structure, bonding, and optical-absorption calculations for the IGF model are most revealing. In addition to providing a logical explanation of the mechanical properties, they add information about the origin of the highly localized states at the top of the VB induced by the defects near the band gap. The similarities in the DOS for the IGF model under strain and the atomspecific partial DOS (PDOS) for atoms in the glassy region give us an overall picture of the electronic structure of the IGF in $\beta-\mathrm{Si}_{3} \mathrm{~N}_{4}$. The rather surprising result is that the presence of the IGF layer has little effect on the anisotropy of the optical absorption (and also the longitudinal sound velocity) of the model. They depend predominately on the orientations of the crystalline planes of the bulk crystal. In polycrystalline ceramics, the orientations of the grains are random and the samples can be considered isotropic. The directionally averaged optical absorption and sound velocity will be good predictions of experimental measurements.

The evaluation of the electrostatic potential difference in the IGF model is significant because it validates the spacecharge model in ceramic materials based on quantummechanical calculations. In interpreting this result, fair caution must be taken for the following reasons: (1) the spacecharge model is usually considered at a much larger length scale than the scale of the IGF model. (2) Different signs can be encountered due to different definitions of the potential and its reference point and (3) there are approximations used in the estimation of the electrostatic potential both in experiments (sample characterization, limited resolutions, normalization procedures, large uncertainty of the IGF width, etc.) and in computation (finite model size, the way the potential is averaged within the IGF, accuracy of the self-consistent field potential, and possible limitations of DFT-LDA). The theoretical values shown here probably represent an upper limit since the IGF region is assumed to be fully dense without any of the porous low-density regions that do exist in real samples. Although we provided quantitative values on the electrostatic potential distribution and its trend under tensile extension, the actual numbers may be less certain depending on the model and other factors. However, such data are difficult to obtain by any other means. In the work by Koch et $a l .{ }^{78} \mathrm{x}$-ray or electron-diffraction techniques were used which depend on the charge density and hence electrostatic potential of the samples. They reported the reconstruction of a one-dimensional potential profile across an IGF from an energy-filtered electron-diffraction pattern. Reference 78 proposed a method but did not provide any quantitative or qualitative data on the electrostatic potential across the IGF. We note that they assumed an average potential value of 17.4 $\mathrm{eV}$ for the bulk crystal and $11.0 \mathrm{eV}$ for $\mathrm{SiO}_{2}$ to imply a 
difference $6.4 \mathrm{eV}$. These assumed values are in reasonable agreement with our calculated values.

The breaking of the IGF under tensile strain based on large-scale computer experiments provides evidence that cracks can propagate through the glassy region relatively easily and is consistent with experimental observations ${ }^{16,79}$ The graphic presentations of strain field evolution provide the necessary insight on the fracture behavior of the IGF model and its relation to the electronic structure. The most interesting part is the formation of surfacial films which are the surface analog of the IGF found within bulk polycrystalline material. ${ }^{8,9}$ Such behavior has not been previously reported using realistic simulations.

Finally, it is fitting to discuss the implication of our results in consideration of the phase field model which has been quite successful in describing the formation of IGFs in ceramics based on thermodynamic grounds. ${ }^{56,80,81}$ Many of the parameters used in the phase field model to characterize the structure of an IGF (crystal grains, crystal orientation, thickness, density, composition, structure of the films, etc.) are readily available from the $a b$ initio simulations demonstrated in this paper. While a dependence on the thermodynamic variables of temperature and pressure was not part of this study, one could view the tensile experiment as simulating an IGF model with a decreasing density.

\section{CONCLUSIONS}

In summary, we have presented the results of physical properties calculations and a simulation of the failure mechanism of a prismatic-IGF model using $a b$ initio methods. These results provide necessary information for the fundamental understanding of the mechanical behavior and electronic interaction of complex microstructures in ceramic materials. The deformation pattern under tensile strain shows complicated behavior. The postfailure sample resembles a coated surfacial film on bulk $\mathrm{Si}_{3} \mathrm{~N}_{4}$ crystal with its own interesting properties.

The present large-scale ab initio simulations on materials with microstructures are made possible only with the resources provided by advanced supercomputers. It is envisioned that continued improvement of the facility and methodology can lead us to address some of the more challenging problems in the same area following the approach used in the present work. For example, we could use larger and even more realistic IGF models with different $\mathrm{N}$ content and compositional gradients. It is possible to dope the IGF with different rare-earth elements such as $\mathrm{Y}, \mathrm{La}, \mathrm{Yb}$, or $\mathrm{Lu}$; and to see how and why the mechanical properties are changed as observed experimentally. In addition to tensile experiments, compression and shear deformation experiments with multiaxial deformation could be carried out to gain further insights on the deformation behavior of ceramic crystals and the accompanying variations in the electrostatic potential distributions. It is also possible to use the present IGF model to perform atom-by-atom calculation of the x-ray appearance near-edge structure/ELNES (electron energy-loss near-edge structure) spectra (Si-K, N-K, and O-K edges, and doped metal edges) ${ }^{82}$ and to apply the recently developed spectral imaging technique ${ }^{83}$ to visualize in three dimensions the connection between the calculated spectra and the electronic structure. These are important and challenging problems in materials research using ab initio methods that are within our reach.

\section{ACKNOWLEDGMENTS}

This work is supported by the U.S. Department of Energy, Office of Basic Energy Sciences, Division of Materials Science and Engineering under Grant No. DE-FG0284DR45170. This research used the resources of NERSC supported by the Office of Science of DOE under Contract No. DE-AC03-76SF00098. L.O. is supported by DOE under Grant No. DE-FE0000103.
${ }^{1}$ J. Luo, Crit. Rev. Solid State Mater. Sci. 32, 67 (2007).

${ }^{2}$ A. Subramaniam, C. T. Koch, R. M. Cannon, and M. Rühle, Mater. Sci. Eng., A 422, 3 (2006).

${ }^{3} \mathrm{See}$, for example, Grain Boundary Engineering in Ceramics, Ceramic Transitions Vol. 118, edited by T. Sakuma, L. M. Sheppard, and Y. Ikuhara (American Ceramic Society, Columbus, $\mathrm{OH}, 2000)$.

${ }^{4}$ M. J. Hoffmann, in Tailoring of Mechanical Properties of Si3N4 Ceramics, edited by M. J. Hoffmann and G. Petzow (Kluwer Academic, Dordrecht, 1994), pp. 59-72.

${ }^{5}$ M. Rühle, Adv. Mater. 9, 195 (1997).

${ }^{6}$ S. J. Dillon and M. P. Harmer, Acta Mater. 55, 5247 (2007).

${ }^{7}$ S. J. Dillon, M. Tang, W. C. Carter, and M. P. Harmer, Acta Mater. 55, 6208 (2007).

${ }^{8}$ J. Luo and Y.-M. Chiang, J. Eur. Ceram. Soc. 19, 697 (1999).

${ }^{9}$ J. Luo and Y.-M. Chiang, Acta Mater. 48, 4501 (2000).

${ }^{10}$ D. R. Clarke, J. Am. Ceram. Soc. 70, 15 (1987).

${ }^{11}$ D. R. Clarke, T. M. Shaw, A. P. Philipse, and R. G. Horn, J. Am.
Ceram. Soc. 76, 1201 (1993).

${ }^{12}$ J. W. Cahn, J. Chem. Phys. 66, 3667 (1977).

${ }^{13}$ H. D. Ackler and Y.-M. Chiang, in Thin Intergranular Films in Ceramics, edited by A. P. Tomsia and A. Glaeser (Plenum Press, New York, 1998), pp. 149-160.

${ }^{14}$ M. Bobeth, D. R. Clarke, and W. Pompe, J. Am. Ceram. Soc. 82, 1537 (1999).

15 J. W. Cahn and J. E. Hilliard, J. Chem. Phys. 28, 258 (1958).

${ }^{16}$ R. L. Satet, M. J. Hoffmann, and R. M. Cannon, Mater. Sci. Eng., A 422, 66 (2006).

${ }^{17}$ H.-J. Kleebe, M. K. Cinibulk, R. M. Cannon, and M. Rühle, J. Am. Ceram. Soc. 76, 1969 (1993).

${ }^{18}$ I. Tanaka, H.-J. Kleebe, M. K. Cinibulk, J. Bruley, D. R. Clarke, and M. Rühle, J. Am. Ceram. Soc. 77, 911 (1994).

${ }^{19}$ X. Pan, H. Gu, R. van Weeren, S. C. Danforth, R. M. Cannon, and M. Rühle, J. Am. Ceram. Soc. 79, 2313 (1996).

${ }^{20}$ C. M. Wang, X. Pan, M. J. Hohhman, R. M. Cannon, and M. Rühle, J. Am. Ceram. Soc. 79, 788 (1996). 
${ }^{21}$ H. Gu, R. M. Cannon, and M. Rühle, J. Mater. Res. 13, 376 (1998).

${ }^{22}$ R. M. Cannon, M. Rühle, M. J. Hoffmann, R. H. French, H. Gu, A. P. Tomsia, and E. Saiz, Ceram. Trans. 118, 427 (2000).

${ }^{23}$ N. Shibata, S. J. Pennycook, T. R. Gosnell, G. S. Painter, W. A. Shelton, and P. F. Becher, Nature (London) 428, 730 (2004).

${ }^{24}$ A. Ziegler, J. C. Idrobo, M. K. Cinibulk, C. Kisielowski, N. D. Browning, and R. O. Ritchie, Science 306, 1768 (2004).

${ }^{25}$ A. Ziegler, J. C. Idrobo, M. K. Cinibulk, C. Kisielowski, N. D. Browning, and R. O. Ritchie, Appl. Phys. Lett. 88, 041919 (2006).

${ }^{26}$ G. B. Winkelman, C. Dwyer, T. S. Hudson, D. Nguyen-Manh, M. Doblinger, R. L. Satet, M. J. Hoffmann, and D. J. H. Cockayne, Appl. Phys. Lett. 87, 061911 (2005).

${ }^{27}$ W. Walkosz, R. F. Klie, S. Ogut, A. Borisevich, P. F. Becher, S. J. Pennycook, and J. C. Idrobo, Appl. Phys. Lett. 93, 053104 (2008).

${ }^{28}$ M. Yoshiya, K. Tatsumi, I. Tanaka, and H. Adachi, J. Am. Ceram. Soc. 85, 109 (2002).

${ }^{29}$ M. Yoshiya, I. Tanaka, H. Adachi, and R. M. Cannon, Int. J. Mater. Res. 101, 57 (2010).

${ }^{30}$ S. H. Garofalini, Mater. Sci. Eng., A 422, 115 (2006).

${ }^{31}$ S. H. Garofalini and W. Luo, J. Am. Ceram. Soc. 86, 1741 (2003).

${ }^{32}$ T. S. Hudson, D. Nguyen-Manh, A. C. T. van Duin, and A. P. Sutton, Mater. Sci. Eng., A 422, 123 (2006).

${ }^{33}$ P. Hohenberg and W. Kohn, Phys. Rev. 136, B864 (1964).

${ }^{34}$ W. Kohn and L. J. Sham, Phys. Rev. 140, A1133 (1965).

${ }^{35}$ P. Rulis, J. Chen, L. Ouyang, W. Y. Ching, X. Su, and S. H. Garofalini, Phys. Rev. B 71, 235317 (2005).

${ }^{36}$ J. Chen, L. Ouyang, P. Rulis, A. Misra, and W. Y. Ching, Phys. Rev. Lett. 95, 256103 (2005).

${ }^{37}$ W. Y. Ching, Jun Chen, Paul Rulis, Lizhi Ouyang, and Anil Misra, J. Mater. Sci. 41, 5061 (2006).

${ }^{38}$ A. Misra, L. Ouyang, J. Chen, and W. Y. Ching, Philos. Mag. 87, 3839 (2007).

${ }^{39}$ P. F. Becher, G. S. Painter, E. Y. Sun, C.-H. Hsueh, and M. J. Lance, Acta Mater. 48, 4493 (2000).

${ }^{40}$ G. S. Painter, P. F. Becher, H. J. Kleebe, and G. Pezzotti, Phys. Rev. B 65, 064113 (2002).

${ }^{41}$ P. F. Becher, N. Shibata, G. S. Painter, F. Averill, K. van Benthem, H.-T. Lin, and S. B. Walters, J. Am. Ceram. Soc. 93, 570 (2010).

${ }^{42}$ G. S. Painter, F. W. Averill, P. F. Becher, N. Shibata, L. v. Benthem, and S. J. Penneycook, Phys. Rev. B 78, 214206 (2008).

${ }^{43}$ G. S. Painter, P. F. Becher, W. A. Shelton, R. L. Satet, and M. J. Hoffmann, Phys. Rev. B 70, 144108 (2004).

${ }^{44}$ W. Y. Ching, P. Rulis, L. Ouyang, and A. Misra, Appl. Phys. Lett. 94, 051907 (2009).

${ }^{45}$ G. Kresse and J. Hafner, Phys. Rev. B 47, 558 (1993).

${ }^{46}$ G. Kresse and J. Furthmüller, Comput. Mater. Sci. 6, 15 (1996); Phys. Rev. B 54, 11169 (1996).

${ }^{47}$ W. Y. Ching, J. Am. Ceram. Soc. 73, 3135 (1990).

${ }^{48}$ A. R. Oganov, J. Chen, C. Gatti, Yanzhang Ma, Yanming Ma, C. W. Glass, Z. Liu, T. Yu, O. O. Kurakevych, and V. L. Solozhenko, Nature (London) 457, 863 (2009).

${ }^{49}$ D. Vanderbilt, Phys. Rev. B 41, 7892 (1990).

${ }^{50}$ R. S. Mulliken, J. Chem. Phys. 23, 1841 (1955).

${ }^{51} \mathrm{~K}$. Refson, MOLDY package available at http://www.earth.ox.uk/ $\sim$ heith/mody.html
${ }^{52}$ J. M. Soler, E. Artacho, J. D. Gale, A. Garcia, J. Junquera, P. Orderjon, and D. Sanchez-Portal, J. Phys.: Condens. Matter 14, 2745 (2002).

${ }^{53}$ G. B. Winkelman, C. Dwyer, C. Marsh, T. S. Hudson, D. Nguyen-Manh, M. Doblinger, and D. J. H. Cockayne, Mater. Sci. Eng., A 422, 77 (2006).

${ }^{54}$ W. Y. Ching and P. Rulis, Phys. Rev. B 77, 035125 (2008).

${ }^{55}$ M. Tang, W. C. Carter, and R. M. Cannon, Phys. Rev. B 73, 024102 (2006)

${ }^{56}$ H. Yao, L. Ouyang and W. Y. Ching, J. Am. Ceram. Soc. 90, 3194 (2007).

${ }^{57}$ R. Hill, Proc. Phys. Soc. London 65, 349 (1951).

${ }^{58}$ W. Y. Ching, L. Ouyang, P. Rulis, and H. Yao, Phys. Rev. B 78, 014106 (2008).

${ }^{59}$ W. Y. Ching, Paul Rulis, and A. Misra, Acta Biomater. 5, 3067 (2009).

${ }^{60}$ R. Vogelgesang, M. Grimsdich, and J. S. Wallace, Appl. Phys. Lett. 76, 982 (2000).

${ }^{61}$ P. Kroll, J. Non-Cryst. Solids 351, 1127 (2005).

${ }^{62}$ E. Robert Newnham, Properties of Materials, Anisotropy, Symmetry, Structure (Oxford University Press, Oxford, 2005).

${ }^{63}$ M.-Z. Huang, L. Ouyang, and W. Y. Ching, Phys. Rev. B 59, 3540 (1999).

${ }^{64}$ P. W. Anderson, Phys. Rev. 109, 1492 (1958).

${ }^{65}$ Y.-N. Xu and W. Y. Ching, Phys. Rev. B 51, 17379 (1995).

${ }^{66}$ K. Lehovec, J. Chem. Phys. 21, 1123 (1953).

${ }^{67}$ K. L. Kliewer and J. S. Koehler, Phys. Rev. 140, A1226 (1965); Phys. Rev. 140, A1241 (1965).

${ }^{68}$ K. L. Kliewer, J. Phys. Chem. Solids 27, 705 (1966); 27, 719 (1966).

${ }^{69}$ M. F. Yan, R. M. Cannon, and K. H. Bowen, J. Appl. Phys. 54, 764 (1983).

${ }^{70}$ J. A. S. Ikeda and Y.-M. Chiang, J. Am. Ceram. Soc. 76, 2437 (1993).

${ }^{71}$ J. A. S. Ikeda, Y.-M. Chiang, A. J. Garratt-Reed, and J. B. Vander Sande, J. Am. Ceram. Soc. 76, 2447 (1993).

${ }^{72}$ F. Lofaj and S. M. Wiederhorn, J. Ceram. Proc. Res. 10, 269 (2009).

${ }^{73}$ J. J. Melendiz-Martinez and A. Domiguez-Rodriguez, Prog. Mater. Sci. 49, 190107 (2004).

${ }^{74}$ J. Chen, Y.-N. Xu, P. Rulis, L. Ouyang, and W. Y. Ching, Acta Mater. 53, 403 (2005).

${ }^{75}$ S. Ogata, Y. Umeno, and M. Kohyama, Modell. Simul. Mater. Sci. Eng. 17, 013001 (2009).

${ }^{76}$ S. Ogata, N. Horosaki, C. Kocer, and H. Kitagawa, Phys. Rev. B 64, 172102 (2001).

${ }^{77}$ N. Hirosaki, C. Kocer, S. Ogata, and K. Tasumi, Phys. Rev. B 71, 104105 (2005).

${ }^{78}$ C. T. Koch, S. Bhattacharyya, M. Rühle, R. L. Satet, and M. J. Hoffmann, Microsc. Microanal. 12, 160 (2006).

${ }^{79}$ S. Ii, C. Iwamoto, K. Matsunga, T. Yamamoto, M. Yoshiya, and Y. Ikuhara, Philos. Mag. 84, 2767 (2004).

${ }^{80}$ R. Kobayashi, J. A. Warren, and M. C. Carter, J. Phys. D 119, 415 (1998).

${ }^{81}$ C. M. Bishop, M. Tang, R. M. Cannon, and W. C. Carter, Mater. Sci. Eng., A 422, 102 (2006).

${ }^{82}$ W. Y. Ching and P. Rulis, J. Phys.: Condens. Matter 21, 104202 (2009).

${ }^{83}$ P. Rulis, A. R. Rupini, S. J. Pennycook, and W. Y. Ching, Ultramicroscopy 109, 1472 (2009). 